International Journal of Pure and Applied Mathematics

Volume 105 No. 4 2015, 669-683

ISSN: 1311-8080 (printed version); ISSN: 1314-3395 (on-line version)

url: http://www.ijpam.eu

doi: http://dx.doi.org/10.12732/ijpam.v105i4.8

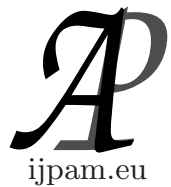

\title{
EPIPOLAR GEOMETRY WITH A FUNDAMENTAL MATRIX IN CANONICAL FORM
}

\author{
Georgi Hristov Georgiev ${ }^{1}$, Vencislav Dakov Radulov² \\ ${ }^{1}$ Faculty of Mathematics and Informatics \\ Konstantin Preslavsky University \\ Shumen, BULGARIA \\ ${ }^{2}$ Department of Descriptive Geometry \\ and Engineering Graphics \\ University of Architecture \\ Civil Engineering and Geodesy \\ Sofia, BULGARIA
}

\begin{abstract}
Epipolar geometry is a main tool in Photogrammetry and Computer Vision. In the paper, we investigate the relations between Epipolar geometry with finite epipolar points and its fundamental matrix with respect to affine coordinate systems. We examine different mutual position of the projection planes and the corresponding canonical form of the fundamental matrix. The case of parallel projection planes is presented from practical point of view.
\end{abstract}

AMS Subject Classification: 51M15, 51N05, 68U05

Key Words: epipolar geometry, fundamental matrix, correlation between projection planes

\section{Introduction}

The reconstruction of a $3 \mathrm{D}$ object from two or more images is a basic problem in Photogrammetry, Computer Vision and Robotics. Epipolar geometry has been a fundamental tool for its solution in the last two decades. Epipolar

Received: August 14, 2015

(C) 2015 Academic Publications, Ltd.

${ }^{\S}$ Correspondence author url: www.acadpubl.eu 
geometry describes the geometrical relationship between two perspective views of one and the same 3D scene. The first step to a reconstruction is estimating the fundamental matrix. The fundamental $3 \times 3$-matrix $\mathbf{F}$ is the algebraic representation of epipolar geometry. If $M \in \omega$ and $M^{\prime} \in \omega^{\prime}$ form a pair of corresponding points and their homogeneous coordinates (written as vectorcolumns) with respect to plane coordinate systems $\mathcal{B}=O x y$ in $\omega$ and $\mathcal{B}^{\prime}=$ $O^{\prime} x^{\prime} y^{\prime}$ in $\omega^{\prime}$ are $\mathbf{p}=\left(x_{M}, y_{M}, 1\right)^{T}$ and $\mathbf{p}^{\prime}=\left(x_{M^{\prime}}^{\prime}, y_{M^{\prime}}^{\prime}, 1\right)^{T}$, respectively, then

$$
\left(\mathbf{p}^{\prime}\right)^{T} \mathbf{F} \mathbf{p}=0
$$

The matrix $\mathbf{F}$ has a rank 2, in particular $\operatorname{det}(\mathbf{F})=0$, and 7 degrees of freedom. If at least 8 pairs of corresponding points are known, then it is possible by linear methods to solve (1) for the entries of $\mathbf{F}$ up to scale. A non-linear solution is available for 7 pairs. If more than 8 pairs are given, then a least-squares solution can be found. This is the general principle of the methods for computing the fundamental matrix. The 8-point algorithm (the normalized 8-point algorithm) is described in [4], [7], [11]. A new eight-point algorithm is presented in [12]. The fundamental matrix is computed by parametrization in [4], [7], [10], [11], and by the use of the projection matrices in [4], [7], [11]. This is generalized in [8] by the Grassmann tensor. Another compact algorithm for computing the fundamental matrix is presented in [9]. A function from the Open Source Computer Vision Library is used for computation of the fundamental matrix in [3]. An affine fundamental matrix obtained by the correspondence of a pair of ellipses is introduced in [1]. All these methods use measured coordinates of the image points.

In this paper, we obtain the simplest form of the fundamental matrix called a canonical form when the epipolar points are finite. We look for this form over $\mathbb{Z}$ with minimal Frobenius norm. Using the canonical form of the fundamental matrix we can rectify the measured coordinates of the image points by a minimization of the distance between the image points and their corresponding epipolar lines. Similar results for the epipolar geometry with one epipolar point at infinity can be found in [5].

The paper is organized as follows. The next section contains some basic definitions and facts about the epipolar geometry. The main result for the canonical form of the fundamental matrix is proved in Section 3. Geometrical and algebraic constructions for the canonical bases in the projection planes are given in Sections 4 and 5. The last section describes a possible applications of the canonical form of the fundamental matrix. 


\section{Epipolar Geometry}

Let us consider two central projections $\Pi$ and $\Pi^{\prime}$ in the Euclidean space $\mathbb{E}^{3}$ determined respectively by the pairs $(\bar{S}, \omega)$ and $\left(\overline{S^{\prime}}, \omega^{\prime}\right)$, where $\omega \subset \mathbb{E}^{3}, \omega^{\prime} \subset \mathbb{E}^{3}$ are the projection planes, and $\bar{S} \in \mathbb{E}^{3} \backslash\{\omega\}, \overline{S^{\prime}} \in \mathbb{E}^{3} \backslash\left\{\omega^{\prime}\right\}$ are the projection (or perspective) centers. We can associate to the projection $\Pi$ (respectively $\Pi^{\prime}$ ) a Cartesian coordinate system $\overline{\mathcal{B}}$ (respectively $\overline{\mathcal{B}^{\prime}}$ ) called a camera frame. The origin of $\overline{\mathcal{B}}$ (respectively $\overline{\mathcal{B}^{\prime}}$ ) is placed at the projection center $\bar{S}$ (respectively $\left.\overline{S^{\prime}}\right)$. The optical ray of the camera coincides with the $\bar{z}\left(\overline{z^{\prime}}\right)$ axis and the axes $\bar{x}, \bar{y}\left(\overline{x^{\prime}}, \overline{y^{\prime}}\right)$ are parallel to the projection plane. The point $H=z \bigcap \omega\left(H^{\prime}=\right.$ $\left.z^{\prime} \cap \omega^{\prime}\right)$ is called a principal point. Then, distance $(\bar{S}, \omega)=\operatorname{distance}(\bar{S}, H)=d$ (distance $\left.\left(\overline{S^{\prime}}, \omega^{\prime}\right)=\operatorname{distance}\left(\overline{S^{\prime}}, H^{\prime}\right)=d^{\prime}\right)$ is a focal length. Furthermore, we

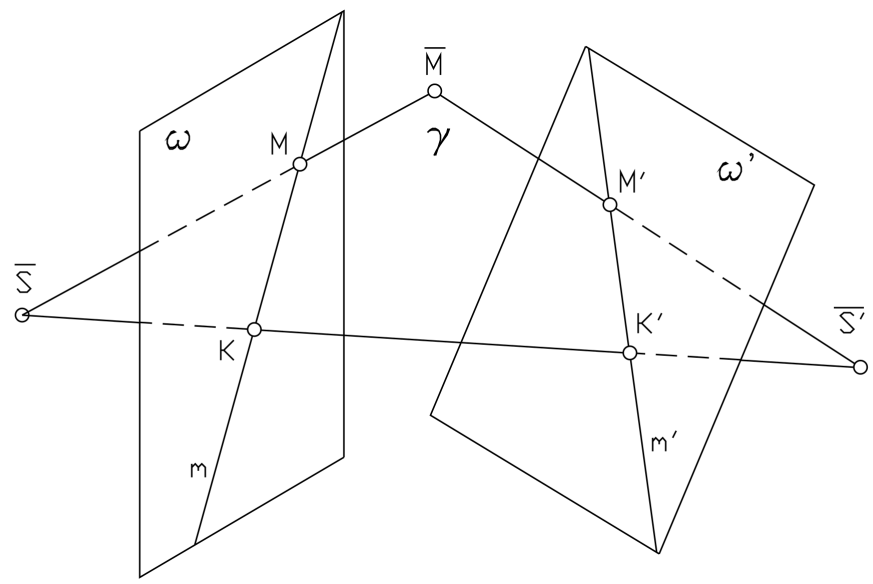

Figure 1: Epipolar geometry

define a plane coordinate system $\mathcal{B}$ (respectively $\mathcal{B}^{\prime}$ ) called an image frame in the projection plane $\omega$ (respectively $\omega^{\prime}$ ). Its origin is placed at $H$ (respectively $H^{\prime}$ ) and the axes are parallel to the axes of the camera frame.

- The joining line $\bar{S} \vee \overline{S^{\prime}}$ is called a baseline (see Fig. 1).

- The epipole (or epipolar point) is the intersection point of the line joining the perspective centers with the image plane. There are two epipolar points $K=\left(\bar{S} \vee \overline{S^{\prime}}\right) \bigcap \omega$ and $K^{\prime}=\left(\bar{S} \vee \overline{S^{\prime}}\right) \bigcap \omega^{\prime}$.

- An epipolar plane is a plane containing the baseline.

- An epipolar line is the intersection of an epipolar plane with the image plane. All epipolar lines intersect at the epipole.

Let $\bar{M} \in \mathbb{E}^{3}$ be an arbitrary space point, different from $\bar{S}$ and $\overline{S^{\prime}}$, and let the 
points $M=(\bar{S} \vee \bar{M}) \bigcap \omega, M^{\prime}=\left(\overline{S^{\prime}} \vee \bar{M}\right) \bigcap \omega^{\prime}$ be its central projections (see Fig. $1)$. Then, the pair $\left(M, M^{\prime}\right)$ is called a pair of corresponding points. If $M \in \omega$ is an arbitrary point different from $K$, then the epipolar plane $\gamma$ passing through the points $\bar{S}, \overline{S^{\prime}}, M$ intersects the projection plane $\omega^{\prime}$ in the epipolar line $m^{\prime}$, or shortly $m^{\prime}=\gamma \bigcap \omega^{\prime}$. In this way, there is a correlation $\mathcal{F}: \omega \rightarrow \omega^{\prime}$ which maps an arbitrary point $M \in \omega \backslash\{K\}$ to its epipolar line $m^{\prime}=M^{\prime} \vee K^{\prime} \subset \omega^{\prime}$, i.e. $M \stackrel{\mathcal{F}}{\rightarrow} m^{\prime}$. The fundamental matrix $\mathbf{F}$ is an algebraic representation of this correlation with respect to given affine coordinate systems $\mathcal{B}=O x y$ in $\omega$ and $\mathcal{B}^{\prime}=O^{\prime} x^{\prime} y^{\prime}$ in $\omega^{\prime}$. This means that if the coordinate vector $\mathbf{p}=\left(x_{M}, y_{M}, 1\right)^{T}$ (respectively, $\mathbf{p}^{\prime}=\left(x_{M^{\prime}}^{\prime}, y_{M^{\prime}}^{\prime}, 1\right)^{T}$ ) represents homogeneous coordinates of the point $M \in \omega$ (respectively $M^{\prime} \in \omega^{\prime}$ ) with reference to $\mathcal{B}$ (respectively $\mathcal{B}^{\prime}$ ), and if $m_{1} x+m_{2} y+m_{3}=0$ (respectively $m_{1}^{\prime} x^{\prime}+m_{2}^{\prime} y^{\prime}+m_{3}^{\prime}=0$ ) is the equation of $m$ (respectively $m^{\prime}$ ) with reference to $\mathcal{B}$ (respectively $\mathcal{B}^{\prime}$ ), then

$$
\mathbf{F} \mathbf{p}=\left(m_{1}^{\prime}, m_{2}^{\prime}, m_{3}^{\prime}\right)^{T} \quad \text { and } \quad \mathbf{F}^{T} \mathbf{p}^{\prime}=\left(m_{1}, m_{2}, m_{3}\right)^{T}
$$

Moreover, if the vector $\mathbf{k}=\left(x_{K}, y_{K}, 1\right)^{T}\left(\right.$ resp. $\left.\mathbf{k}^{\prime}=\left(x_{K^{\prime}}^{\prime}, y_{K^{\prime}}^{\prime}, 1\right)^{T}\right)$ represents homogeneous coordinates of the epipolar point $K \in \omega\left(\right.$ resp. $\left.K^{\prime} \in \omega^{\prime}\right)$ with reference to $\mathcal{B}\left(\operatorname{resp} . \mathcal{B}^{\prime}\right)$, then

$$
\mathbf{F} \mathbf{k}=(0,0,0)^{T} \text { and } \quad \mathbf{F}^{T} \mathbf{k}^{\prime}=(0,0,0)^{T} .
$$

(See for details [4], [7], [11]). The use of homogeneous coordinates in the projection planes $\omega$ and $\omega^{\prime}$ allow to consider any projection plane as a projective extension of the Euclidean plane. Thus, any finite or Euclidean point in $\omega$ and $\omega^{\prime}$ has nonzero third homogeneous coordinate while the third homogeneous coordinate of any point at infinity is always equal to 0 . Moreover at least one of the three homogeneous coordinates of any finite point and any point at infinity is different from 0. Some recent applications of epipolar geometry can be found in $([2])$ and $[6]$.

\section{Canonical Form of the Fundamental Matrix}

The coordinates of the points and the elements of the fundamental matrix in (1) depend on the choice of coordinate systems in the projection planes. In this section, we introduce special affine coordinate systems in the projection planes $\omega$ and $\omega^{\prime}$. The fundamental matrix with respect to these coordinate systems has only integer elements and a minimal Frobenius norm. 
Theorem 1. Let $\Pi=(\bar{S}, \omega)$ and $\Pi^{\prime}=\left(\overline{S^{\prime}}, \omega^{\prime}\right)$ be two central projections determining epipolar geometry. Then the two epipolar points are finite if and only if there exist plane coordinate systems $\mathcal{B}_{C}=O x y$ in $\omega$ and $\mathcal{B}_{C}^{\prime}=O^{\prime} x^{\prime} y^{\prime}$ in $\omega^{\prime}$ such that the fundamental matrix $\mathbf{F}$ (up to scale) with respect to $\mathcal{B}_{C}$ and $\mathcal{B}_{C}^{\prime}$ is equal to

$$
\left[\begin{array}{rrl}
0 & 1 & 0 \\
-1 & 0 & 0 \\
0 & 0 & 0
\end{array}\right] \text {. }
$$

Proof. First, we assume that the baseline $\bar{S} \vee \overline{S^{\prime}}$ intersects the two projection planes at two finite (or Euclidean) epipolar points $K \in \omega$ and $K^{\prime} \in \omega^{\prime}$. Then, we introduce two coordinate systems $\mathcal{B}_{C}=O x y$ in $\omega$ and $\mathcal{B}_{C}^{\prime}=O^{\prime} x^{\prime} y^{\prime}$ in $\omega^{\prime}$ as follows. The origins coincide with the epipolar points, i.e. $K \equiv O$ and $K^{\prime} \equiv O^{\prime}$. Consider two different epipolar planes $\gamma, \gamma^{\prime}$ passing through $\bar{S} \vee \overline{S^{\prime}}$ and determine the coordinate axes $x=\gamma \bigcap \omega, y=\gamma^{\prime} \cap \omega$ in $\omega, x^{\prime}=\gamma \bigcap \omega^{\prime}$, as well as the coordinate axes $y^{\prime}=\gamma^{\prime} \cap \omega^{\prime}$ in $\omega^{\prime}$. Then, choosing the unit point $E$ of the affine coordinate system $O x y$ on the bisector $l$ of $\angle x O y$ we can obtain a point $E^{\prime} \in \omega^{\prime}$ such that $E$ and $E^{\prime}$ form a pair of corresponding points in the considered epipolar geometry. In fact, if $\varepsilon$ is the epipolar plane passing through $E$ and $\bar{S} \vee \overline{S^{\prime}}$, then $l^{\prime}=\varepsilon \bigcap \omega^{\prime}$ is the epipolar line corresponding to $E$ and $E^{\prime}=\left(E \vee \overline{S^{\prime}}\right) \cap l^{\prime}$, i.e. $\left(E, E^{\prime}\right)$ is a pair of corresponding points regarding to $E \in \omega$. We take the point $E^{\prime}$ as the unit point of the affine coordinate system $O^{\prime} x^{\prime} y^{\prime}$ in $\omega^{\prime}$. Under the above assumptions we compute the fundamental matrix $\mathbf{F}=\left[f_{i j}\right]_{i, j=1,2,3}$. From $\mathbf{k}=(0,0,1)^{T}$ and the left equation in (3) it follows that $f_{13}=f_{23}=f_{33}=0$. In the same way, from $\mathbf{k}^{\prime}=(0,0,1)^{T}$ and the right equation in (3) it follows that $f_{31}=f_{32}=f_{33}=0$. An arbitrary point $A \in x$ with vector-column of homogeneous coordinates $\mathbf{a}=\left(x_{A}, 0,1\right)^{T}$ has a corresponding epipolar line $x^{\prime}$ with an equation $y^{\prime}=0$. Then the left equation of (2) is $\mathbf{F a}=t_{A}(0,1,0)\left(t_{A} \neq 0\right)$, or equivalently $\left(f_{11} x_{A}, f_{21} x_{A}, 0\right)=t_{A}(0,1,0)$ for any $x_{A}$. This means $f_{11}=0$. Similarly, $f_{22}=0$. The unit points $\left(E\right.$ and $\left.E^{\prime}\right)$ of the two coordinate systems form a pair of corresponding points, and the vectorcolumns of their homogeneous coordinates are $\mathbf{e}=(1,1,1)^{T}$ and $\mathbf{e}^{\prime}=(1,1,1)^{T}$, respectively. Applying (1) we get $f_{21}=-f_{12}$. Thus the fundamental matrix up to scale factor can be written in the form (4).

Conversely, let the matrix (4) be the fundamental matrix $\mathbf{F}$ with respect to some affine coordinate systems $\mathcal{B}$ in $\omega$ and $\mathcal{B}^{\prime}$ in $\omega^{\prime}$. Suppose that the homogeneous coordinates of the epipolar point $K$ (resp. $K^{\prime}$ ) are $x_{K}, y_{K}$ and $w_{K}$ (rsp. $x_{K^{\prime}}^{\prime}, y_{K^{\prime}}^{\prime}$ and $\left.w_{K^{\prime}}^{\prime}\right)$. Then, at least one of the real numbers $x_{K}$, $y_{K}, w_{K}$ is different from 0 , and at least one of the real numbers $x_{K^{\prime}}^{\prime}, y_{K^{\prime}}^{\prime}$ and 
$w_{K^{\prime}}^{\prime}$ is different from 0 . In other words, both vectors $\mathbf{k}=\left(x_{K}, y_{K}, w_{K}\right)^{T}$ and $\mathbf{k}^{\prime}=\left(x_{K^{\prime}}^{\prime}, y_{K^{\prime}}^{\prime}, w_{K^{\prime}}^{\prime}\right)^{T}$ are nonzero. Substituting in the left equation in (3) we have $\mathbf{F k}=\left[\begin{array}{rrr}0 & 1 & 0 \\ -1 & 0 & 0 \\ 0 & 0 & 0\end{array}\right]\left(\begin{array}{c}x_{K} \\ y_{K} \\ w_{K}\end{array}\right)=\left(\begin{array}{l}0 \\ 0 \\ 0\end{array}\right)$, i.e. $x_{K}=y_{K}=0$. This implies that $w_{K} \neq 0$, or the epipolar point $K$ is finite. In the same way, from the right equation in (3) it follows that $K^{\prime}$ is a finite point.

Definition 2. The plane coordinate systems $\mathcal{B}_{C}=O x y$ in $\omega$ and $\mathcal{B}_{C}^{\prime}=$ $O^{\prime} x^{\prime} y^{\prime}$ in $\omega^{\prime}$ from Theorem 1 are called canonical bases. The matrix (4) is called a canonical form of the fundamental matrix .

\section{Construction of the Canonical Bases}

There are two approaches for determining the epipolar geometry. The first approach is by two given central projections. The second one is by the fundamental matrix with respect to given coordinate systems in the projection planes. Now, we will present practical ways for obtaining canonical bases in both cases. These ways are based on Theorem 1 and its proof.

\subsection{Epipolar Geometry with Coinciding Projection Planes}

Assume that epipolar geometry is defined by two central projection $\Pi=(\bar{S}, \omega)$ and $\Pi^{\prime}=\left(\overline{S^{\prime}}, \omega^{\prime}\right)$ such that $\omega=\omega^{\prime}, \bar{S} \neq \overline{S^{\prime}}$, and the baseline $\bar{S} \vee \overline{S^{\prime}}$ is not parallel to the plane $\omega$. (see Fig. 2). Then the two epipolar points are coinciding $K \equiv K^{\prime}$. If $\left(M, M^{\prime}\right)$ is a pair of corresponding points, then the line $M \vee M^{\prime}$ is an epipolar line. Every epipolar plane intersects the coinciding projection planes in the coinciding epipolar lines. Let $x \equiv x^{\prime}$ and $y \equiv y^{\prime}$ be two different lines in $\omega \equiv \omega^{\prime}$ passing through $K \equiv K^{\prime}$. Consider a fixed point $E=E^{\prime}$ on the bisector of $\angle x K y$ different from $K$. As in the proof of Theorem 1 it can be shown that the affine coordinate system $\mathcal{B}_{C}=K x y$ in $\omega$ with a unit point $E$ and $\mathcal{B}_{C}^{\prime}=K^{\prime} x^{\prime} y^{\prime}$ in $\omega^{\prime}$ with a unit point $E^{\prime}$ are canonical bases of the considered epipolar geometry.

\subsection{Epipolar Geometry with Intersecting Projection Planes}

Assume that epipolar geometry is defined by two central projection $\Pi=(\bar{S}, \omega)$ and $\Pi^{\prime}=\left(\overline{S^{\prime}}, \omega^{\prime}\right)$ such that $\omega \neq \omega^{\prime}, \omega \vee \omega^{\prime}, \bar{S} \neq \overline{S^{\prime}}$, and the baseline $\bar{S} \vee \overline{S^{\prime}}$ is not 


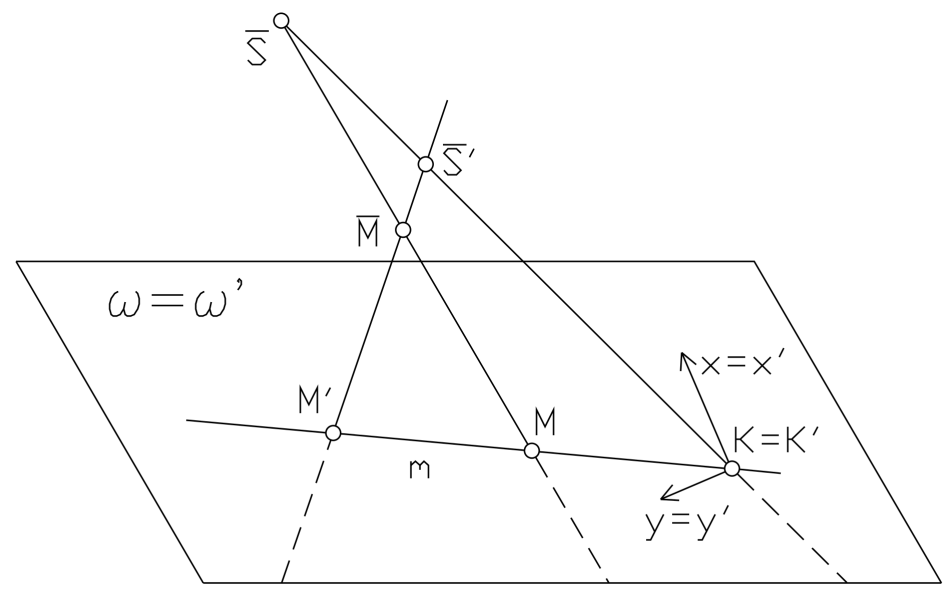

Figure 2: Coinciding projection planes
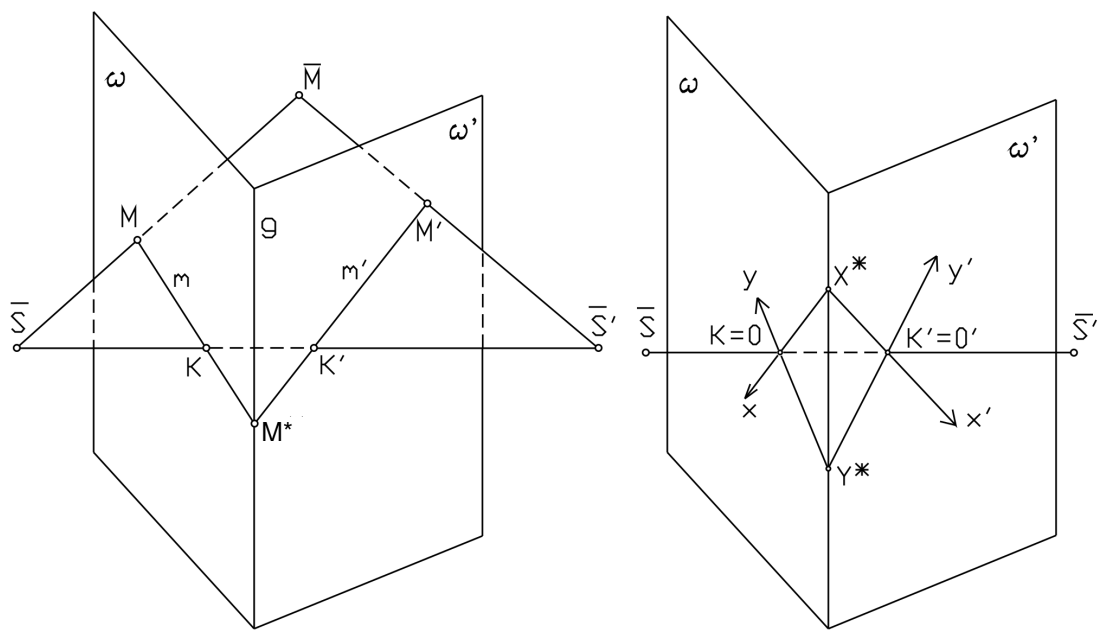

Figure 3: Intersecting projection planes (on the left) and a choice of canonical bases (on the right)

parallel to the planes $\omega$ and $\omega^{\prime}$. (see Fig. 3). Then the intersection $\omega \bigcap \omega^{\prime}=g$ is a line, and the epipolar points $K=\left(\bar{S} \vee \overline{S^{\prime}}\right) \bigcap \omega, K^{\prime}=\left(\bar{S} \vee \overline{S^{\prime}}\right) \bigcap \omega^{\prime}$ are finite. Let $\bar{M} \in \mathbb{E}^{3} \backslash\left\{\left(\bar{S} \vee \overline{S^{\prime}}\right)\right\}$ be an arbitrary point, and let $M \in \omega, M^{\prime} \in \omega^{\prime}$ be its projections. Consider the epipolar plane $\mu$ passing through $\bar{M}, M, M^{\prime}$ and the intersecting point $M^{*}=g \bigcap \mu$. Then, the epipolar line $m=\mu \bigcap \omega$ meets 
the epipolar line $m^{\prime}=\mu \bigcap \omega^{\prime}$ at the point $M^{*}$, or shortly $m \bigcap m^{\prime}=M^{*}$. This implies that the correlation $\mathcal{F}: \omega \rightarrow \omega^{\prime}$ maps an arbitrary point $M \in \omega \backslash\{K\}$ to its epipolar line $m^{\prime}=M^{*} K^{\prime}=M^{\prime} K^{\prime} \subset \omega^{\prime}$, where $M^{*}=(M \vee K) \bigcap g$. The above observations allow is to describe a short procedure for obtaining canonical bases in this particular case.

Input: Epipolar geometry with intersecting projection planes $\omega \bigcap \omega^{\prime}=g$.

1. Find the epipolar points $K$ and $K^{\prime}$.

2. Identify $O=K$ and $O^{\prime}=K^{\prime}$.

3. Choose two different lines $x \subset \omega, y \subset \omega$ passing through $O$ and find the intersecting points $X^{*}=g \bigcap x, Y^{*}=g \bigcap y$.

4. Determine the joining lines $x^{\prime}=X^{*} \vee K^{\prime}$ and $y^{\prime}=Y^{*} \vee K^{\prime}$.

5. Choose a point $E(E \neq O)$ on the bisector of the angle $\angle x O y$ and obtain (as in the proof in Theorem 1) the point $E^{\prime} \in \omega^{\prime}$ such that $\left(E, E^{\prime}\right)$ to be a pair of corresponding points.

Output: Two canonical basis are $\mathcal{B}_{C}=O x y$ with a unit point $E$ in $\omega$ and $\mathcal{B}_{C}^{\prime}=O^{\prime} x^{\prime} y^{\prime}$ with a unit point $E^{\prime}$ in $\omega^{\prime}$.

\subsection{Epipolar Geometry with Parallel Projection Planes}

Assume that epipolar geometry is defined by two central projection $\Pi=(\bar{S}, \omega)$ and $\Pi^{\prime}=\left(\overline{S^{\prime}}, \omega^{\prime}\right)$ such that $\omega \neq \omega^{\prime}, \omega \| \omega^{\prime}, \bar{S} \neq \overline{S^{\prime}}$, and the baseline $\bar{S} \vee \overline{S^{\prime}}$ is not parallel to the plane $\omega$ (see Fig. 4). Then the epipolar points $K=\left(\bar{S} \vee \overline{S^{\prime}}\right) \cap \omega$, $K^{\prime}=\left(\bar{S} \vee \overline{S^{\prime}}\right) \cap \omega^{\prime}$ are finite. If $\left(M, M^{\prime}\right)$ is an arbitrary pair of corresponding points, then the epipolar lines $m=K M$ and $m^{\prime}=K^{\prime} M^{\prime}$ are parallel. Let $\xi$ and $\eta$ be two different planes passing through the baseline and let $\varepsilon$ be a bisector plane of the dihedral angle $\angle(\xi, \eta)$. Consider the intersecting lines $x=\omega \bigcap \xi$, $y=\omega \bigcap \eta, l=\omega \bigcap \varepsilon, x^{\prime}=\omega^{\prime} \bigcap \xi, y^{\prime}=\omega^{\prime} \bigcap \eta, l^{\prime}=\omega^{\prime} \bigcap \varepsilon$. Then we choose a point $E \in l(E \neq K)$ and get the intersecting point $E^{\prime}=l^{\prime} \cap\left(\overline{S^{\prime}} \vee E\right)$. Identifying $O=K, O^{\prime}=K^{\prime}$ we define the affine coordinate system $\mathcal{B}_{C}=O x y$ with a unit point $E$ in the projection plane $\omega$ and the affine coordinate system $\mathcal{B}_{C}^{\prime}=O^{\prime} x^{\prime} y^{\prime}$ with a unit point $E^{\prime}$ in the projection plane $\omega^{\prime}$. As in the proof of Theorem 1 it can be verified that $\mathcal{B}_{C}$ and $\mathcal{B}_{C}^{\prime}$ are canonical bases for this epipolar geometry with parallel projection planes. 


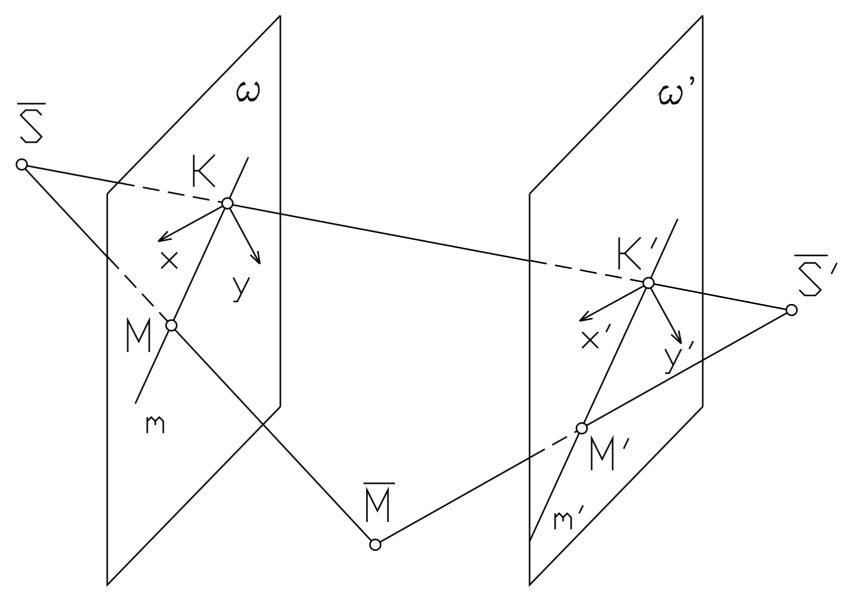

Figure 4: Parallel projection planes

\subsection{Epipolar geometry with a given fundamental matrix}

Assume that epipolar geometry is defined by the fundamental matrix $\mathbf{F}$ with respect to a given coordinate system $\mathcal{B}=O x y$ in $\omega$ and a given coordinate system $\mathcal{B}^{\prime}=O^{\prime} x^{\prime} y^{\prime}$ in $\omega^{\prime}$. Then we can obtain the canonical bases $\mathcal{B}_{C}=$ $O_{C} x_{C} y_{C}$ in $\omega$ and $\mathcal{B}_{C}^{\prime}=O_{C}^{\prime} x_{C}^{\prime} y_{C}^{\prime}$ in $\omega^{\prime}$ in such a way that the elements of $\mathcal{B}_{C}$ are expressed in terms of $\mathcal{B}$ and the elements of $\mathcal{B}_{C}^{\prime}$ are expressed in terms of $\mathcal{B}^{\prime}$. The coordinate vector $\mathbf{k}=\left(k_{1}, k_{2}, k_{3}\right)$ of the epipolar point $K \in \omega$ regarding to $\mathcal{B}$ is a nonzero solution of the equation $\mathbf{F} \mathbf{k}=(0,0,0)^{T}$.

Similarly, the coordinate vector $\mathbf{k}^{\prime}=\left(k_{1}^{\prime}, k_{2}^{\prime}, k_{3}^{\prime}\right)$ of the epipolar point $K^{\prime} \in \omega^{\prime}$ regarding to $\mathcal{B}^{\prime}$ is a nonzero solution of the equation $\mathbf{F}^{T} \mathbf{k}^{\prime}=(0,0,0)^{T}$. If $k_{3} \neq 0$ and $k_{3}^{\prime} \neq 0$ we identify $O_{C}=K$ and $O_{C}^{\prime}=K^{\prime}$. Consider the points $A \in \omega$ with coordinate vector $\left(x_{A}, y_{A}, 1\right)$ and $B \in \omega$ with coordinate vector $\left(x_{B}, y_{B}, 1\right)$ such that the three points $O_{C}, A, B$ are non-collinear and $O_{C}$ is equidistant from $A$ and $B$. Then we denote by $E$ a point in $\omega$ with a coordinate vector $\mathbf{e}=\left(\left(x_{A}+x_{B}\right),\left(y_{A}+y_{B}\right), 1\right)$. Thus, it is defined an affine coordinate system $\mathcal{B}_{C}=O_{C} x_{C} y_{C}$ in $\omega$ with an origin $O_{C}$, axes $x_{C}=O_{C} \vee A, y_{C}=O_{C} \vee B$, and an unit point $E$. Furthermore, if $\mathbf{F a}=\left(a_{1}, a_{2}, a_{3}\right)^{T}$ and $\mathbf{F b}=\left(b_{1}, b_{2}, b_{3}\right)^{T}$, we examine the line $x_{C}^{\prime} \subset \omega^{\prime}$ with equation $a_{1} x^{\prime}+a_{2} y^{\prime}+a_{3}=0$ regarded to $\mathcal{B}^{\prime}$ and the line $y_{C}^{\prime} \subset \omega^{\prime}$ with equation $b_{1} x^{\prime}+b_{2} y^{\prime}+b_{3}=0$ regarded to $\mathcal{B}^{\prime}$, respectively. Let $E_{C}^{\prime} \in \omega^{\prime}$ be a point whose coordinate vector $\mathbf{e}^{\prime}$ is a nonzero solution of the equation $\left(\mathbf{e}^{\prime}\right)^{T} \mathbf{F} \mathbf{e}=0$. Then, it is defined an affine coordinate system $\mathcal{B}_{C}^{\prime}=O_{C}^{\prime} x_{C}^{\prime} y_{C}^{\prime}$ in $\omega^{\prime}$ with a unit point $E_{C}^{\prime}$. From the proof of Theorem 1 it 
follows that the obtained coordinate systems $\mathcal{B}_{C}=O_{C} x_{C} y_{C}$ and $\mathcal{B}_{C}^{\prime}=O_{C}^{\prime} x_{C}^{\prime} y_{C}^{\prime}$ are canonical bases of the epipolar geometry defined by the fundamental matrix F.

\section{A Fundamental Matrix with respect to Special Coordinate Systems}

There is a direct way to define epipolar geometry by fundamental matrix with respect to special affine coordinate systems in the projection planes. Let $O$, $M_{1}, M_{2}, M_{3}$ be four points in general position in $\omega$ (every three points are noncollinear), and let $O^{\prime}, M_{1}^{\prime}, M_{2}^{\prime}, M_{3}^{\prime}$ be also four points in general position in $\omega^{\prime}$. Then there exist epipolar geometry with epipolar points $O, O^{\prime}$ and two pairs of corresponding points $\left(M_{i}, M_{i}^{\prime}\right), i=1,2$. In fact, if $O x y\left(x=O \vee M_{1}, y=\right.$ $\left.O \vee M_{2}\right)$ is an affine coordinate system in $\omega$ with a unit point $M_{3}$, and if $O^{\prime} x^{\prime} y^{\prime}$ $\left(x^{\prime}=O^{\prime} \vee M_{1}^{\prime}, y^{\prime}=O^{\prime} \vee M_{2}^{\prime}\right)$ is an affine coordinate system in $\omega^{\prime}$ with a unit point $M_{3}^{\prime}$, then as in the proof of Theorem 1 we can see that the fundamental matrix (up to scale) with respect of these two coordinate systems is given by

$$
\mathbf{F}=\left[\begin{array}{lll}
0 & 1 & 0 \\
p & 0 & 0 \\
0 & 0 & 0
\end{array}\right], p \neq 0
$$

Moreover $p=-1$ if and only if $\left(M_{3}, M_{3}^{\prime}\right)$ is a pair of corresponding points. Now we examine an important particular case of parallel projection planes.

Theorem 3. Let $\omega$ and $\omega^{\prime}$ be two parallel planes in the Euclidean 3-space, Assume that $O, M_{1}, M_{2}$ are three non-collinear points in $\omega, O^{\prime}, M_{1}^{\prime}, M_{2}^{\prime}$ are three non-collinear points in $\omega^{\prime}$, and $O \vee M_{i} \| O^{\prime} \vee M_{i}{ }^{\prime}(i=1,2)$. Then, there exists an epipolar geometry with projection planes $\omega$ and $\omega^{\prime}$, epipolar points $O$ and $O^{\prime}$, and two pairs of corresponding points $\left(M_{i}, M_{i}{ }^{\prime}\right), i=1,2$.

Proof. There is a unique affine coordinate system $O x y$ in $\omega$ such that the coordinate vectors of $O, M_{1}$ and $M_{2}$ are $\mathbf{o}=(0,0,1)^{T}, \mathbf{m}_{1}=(1,0,1)^{T}$ and $\mathbf{m}_{2}=(0,1,1)^{T}$, respectively. Similarly, there is a unique affine coordinate system $O^{\prime} x^{\prime} y^{\prime}$ in $\omega^{\prime}$ such that the coordinate vectors of $O^{\prime}, M_{1}^{\prime}$, and $M_{2}^{\prime}$ are $\mathbf{o}^{\prime}=(0,0,1)^{T}, \mathbf{m}_{1}^{\prime}=(1,0,1)^{T}$ and $\mathbf{m}_{2}^{\prime}=(0,1,1)^{T}$, respectively. Let the point $E \in \omega$ with a coordinate vector $\mathbf{e}=(1,1,1)^{T}$ be the unit point of $O x y$. The line $l^{\prime} \subset \omega^{\prime}$ passing through $O^{\prime}$ and parallel to $O \vee E$ has an equation $x^{\prime}+p y^{\prime}=0$ $(p \neq 0)$. Then, there is an epipolar geometry with projection planes $\omega$ and $\omega^{\prime}$ such that its fundamental matrix $\mathbf{F}$ is determined by the matrix equalities 
$\mathbf{F o}=(0,0,0)^{T}, \mathbf{F}^{T} \mathbf{o}^{\prime}=(0,0,0)^{T}, \mathbf{F} \mathbf{m}_{1}=(0,1,0)^{T}, \mathbf{F} \mathbf{m}_{2}=(1,0,0)^{T}, \mathbf{F e}=$ $(1, p, 0)^{T}$. This epipolar geometry has epipolar points $O$ and $O^{\prime}$, two pairs of corresponding points $\left(M_{i}, M_{i}{ }^{\prime}\right), i=1,2$ and its fundamental matrix up to scale is written in the form (5) with respect to $O x y$ and $O^{\prime} x^{\prime} y^{\prime}$.

\section{An Application of the Canonical Form of the Fundamental Matrix}

Let us consider two photographs of one and the same 3D scene such that the second photo is obtained by moving camera along the optical ray without rotation. Then the first position of the camera corresponds to the first photo and determines the projection plane $\omega$, second position of the camera corresponds to the second photo and determines the projection plane $\omega^{\prime}$. Furthermore, we may consider the principal points as the epipolar points of epipolar geometry (see Section 2). Thus, the image frames $\mathcal{B}$ in $\omega$ and $\mathcal{B}^{\prime}$ in $\omega^{\prime}$ (defined also in Section 2) are canonical bases of considered epipolar geometry. In other words, the fundamental matrix with respect to $\mathcal{B}$ and $\mathcal{B}^{\prime}$ is canonical and therefore it can be denoted by $\mathbf{F}_{C}$.

Let us consider a pair of corresponding points $\left(M, M^{\prime}\right)$, i.e. the point $M$ from the first photo and the point $M^{\prime}$ from the second photo represent one and the same 3D point $\bar{M}$. Using coordinate measuring machine we obtain the coordinate vector $\mathbf{m}=(x, y, 1)^{T}$ of $M$ with respect to $\mathcal{B}$ and the coordinate vector $\mathbf{m}^{\prime}=\left(x^{\prime}, y^{\prime}, 1\right)^{T}$ of $M^{\prime}$ with respect to $\mathcal{B}^{\prime}$. Now, we will reduce errors resulting from measurement and distortion in the photos. Let us denote the coordinate vector of the exact coordinates of the point $M$ by $\mathbf{m}_{e}=(u, v, 1)^{T}$ and the coordinate vector of the exact coordinates of the point $M^{\prime}$ by $\mathbf{m}_{e}^{\prime}=\left(u^{\prime}, v^{\prime}, 1\right)^{T}$. Then, $u=x+a, v=y+b, u^{\prime}=x^{\prime}+c, v^{\prime}=y^{\prime}+d$, where the real numbers $a, b, c, d$ are necessary rectifications of the coordinates. From $\left(\mathbf{m}_{e}^{\prime}\right)^{T} \mathbf{F}_{C} \mathbf{m}_{e}^{\prime}=0$ it follows that the numbers $a, b, c, d$ can be found by solving following nonlinear constrained optimization problem:

$$
\begin{gathered}
\min \left\{Q(a, b, c, d)=a^{2}+b^{2}+c^{2}+d^{2}\right\} \\
\text { subject to } \quad\left(\begin{array}{lll}
x^{\prime}+c, & y^{\prime}+d, & 1
\end{array}\right) \mathbf{F}_{C}\left(\begin{array}{c}
x+a \\
y+b \\
1
\end{array}\right)=0 .
\end{gathered}
$$

Using Lagrange multipliers method we get the numbers $a, b, c, d$ satisfying (6) and (7). In this way we obtain the rectifying coordinates $u=x+a, v=$ $y+b, u^{\prime}=x^{\prime}+c, v^{\prime}=y^{\prime}+d$. 
Example 1. Let us consider two photographs of a urban area in Sofia city (Bulgaria) obtained as in the beginning of this section(see Fig. 5 and Fig. 6.). We specify 10 pairs of corresponding points $\left(M_{i}, M_{i}^{\prime}\right), i=1 \ldots 10$ in both photos and measure their Cartesian coordinates with respect to image frames. These coordinates are given in Table 1.

\begin{tabular}{|r|r|r|l|l|r|r|r|l|}
\hline N: & \multicolumn{3}{|c|}{ from the first photo } & & $\mathrm{N}:$ & \multicolumn{3}{|c|}{ from the second photo } \\
\hline 1 & 3.8693 & -24.1966 & 1 & & 1 & -3.0621 & -26.5910 & 1 \\
\hline 2 & -22.6071 & -23.7091 & 1 & & 2 & -29.4471 & -26.3193 & 1 \\
\hline 3 & -6.0136 & -24.6840 & 1 & & 3 & -12.7184 & -26.8627 & 1 \\
\hline 4 & 15.5823 & -13.5953 & 1 & & 4 & 8.7704 & -15.7246 & 1 \\
\hline 5 & -22.4851 & -16.5198 & 1 & & 5 & -29.3382 & -18.9325 & 1 \\
\hline 6 & 10.7019 & -23.7091 & 1 & & 6 & 3.9752 & -26.1923 & 1 \\
\hline 7 & -107.9145 & -10.2075 & 1 & & 7 & -114.6804 & -13.7751 & 1 \\
\hline 8 & -101.8012 & -11.8043 & 1 & & 8 & -109.0391 & -15.3620 & 1 \\
\hline 9 & -121.2696 & 0.0307 & 1 & & 9 & -128.6466 & -3.5976 & 1 \\
\hline 10 & -130.5805 & 7.8268 & 1 & & 10 & -137.9026 & 4.2270 & 1 \\
\hline
\end{tabular}

Table 1: The homogeneous coordinates of the chosen points.

Using the same 10 pairs of points and eight point algorithm we compute up to scale the corresponding fundamental matrix

$$
\mathbf{F}_{\text {eight }}=\left[\begin{array}{rrr}
0.0081 & 0.5687 & 12.4891 \\
-0.5594 & 0.0089 & 5.2196 \\
-13.5618 & -1.0711 & 98.138
\end{array}\right]
$$

We can compare the distances between the point $M_{i}^{\prime}$ and its epipolar line $m_{i}^{\prime}$ obtained by different fundamental matrices and coordinates with different precision. These distances are given in Table 2. In the first column we use the matrix $\mathbf{F}_{\text {eight }}$ and the measured coordinates, in the second column we apply a canonical form of the fundamental matrix $\mathbf{F}_{C}$ and the measured coordinates of $M_{i}$, and in the last column we employ the same canonical matrix $\mathbf{F}_{C}$ and the rectifying coordinates. The numbers in the third column are closest to zero. Thus, we can conclude that the combination of $\mathbf{F}_{C}$ and the rectifying coordinates gives the best results. 


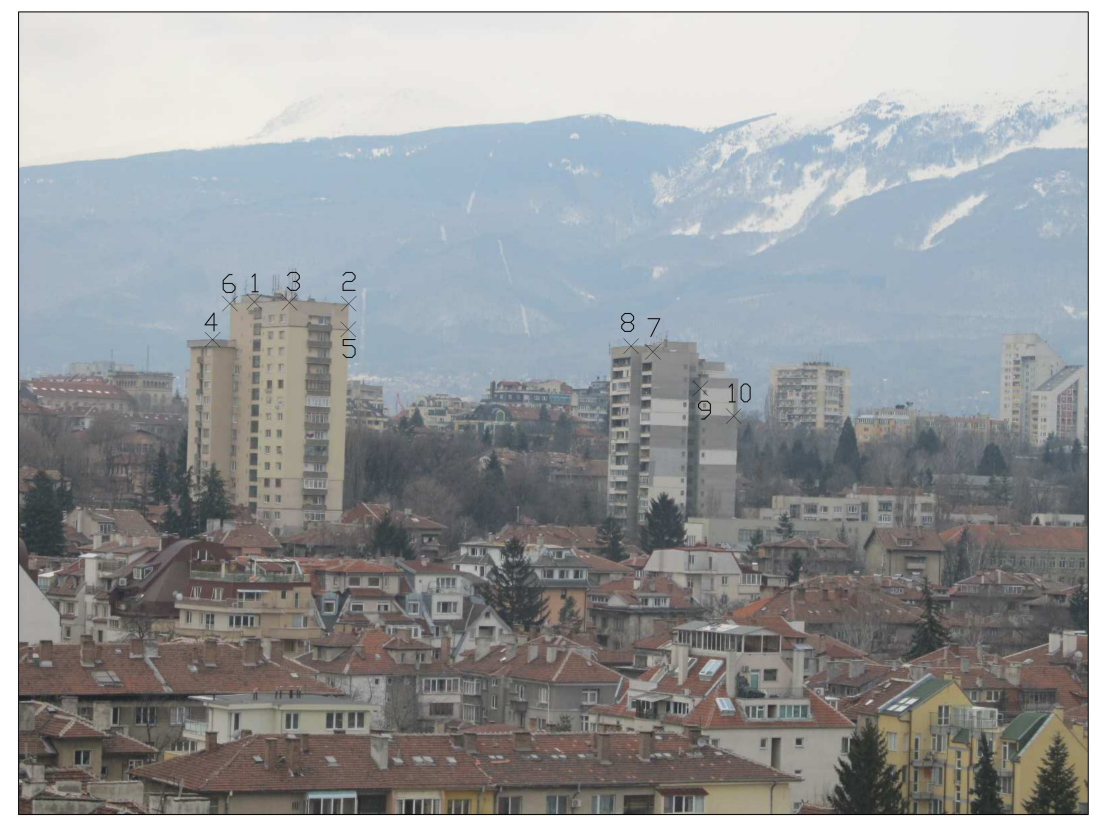

Figure 5: First photo of a urban area

\section{Acknowledgments}

Both authors are partially supported by Konstantin Preslavsky University of Shumen under Grand No. RD-08-281/12.03.2015.

\section{References}

[1] R. Arandjelovic, A. Zisserman, Efficient image retrieval for 3D structures, In: Proceedings of the British Machine Vision Conference, BMVA Press, Durham, UK (2010), 30.1-30.11.

[2] C. Brites, F.Pereira, Epipolar Geometry-based side information creation for multiview WynerZiv video coding, IEEE Transactions on Circuits and Systems for Video Technology 24(10)(2014), 1771 - 1786.

[3] J. Dias, R. Vicente, J. Correia, R. Cruz, Automatic 3D Reconstruction of Urban Areas by using Epipolar Geometry and Template Matching, In: ADETTI/ISCTE, Lisboa, Portugal (2006), 129-138. 


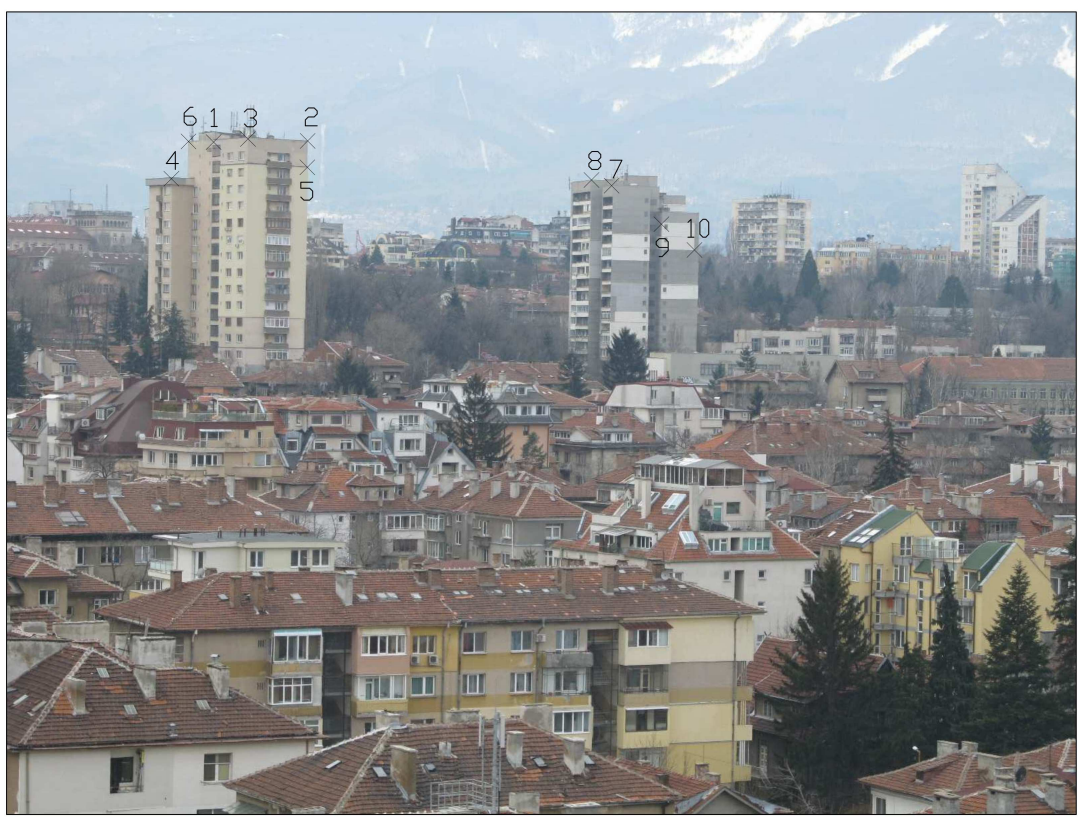

Figure 6: Second photo of a urban area

[4] O. Faugeras, Q. T. Luong, The Geometry of Multiple Images, The MIT Press, Cambridge, Massachusetts (2001).

[5] G. H. Georgiev, V.D. Radulov, Epipolar Geometry with one Epipole at Infinity, American Institute of Physics, Conference Proceedings 1570 (2013), $375-382$.

[6] G. H. Georgiev, V. R. Radulov, A Practical Method for Decomposition of the Essential Matrix, Applied Mathematical Sciences, 8(2014), 8755 -8770.

[7] R. Hartley, A. Zisserman, Multiple View geometry in Computer Vision (Second edition), Cambridge University Press, Cambridge, UK (2003).

[8] R. Hartley, F. Schaffalitzky, Reconstruction from Projections Using Grassmann Tensors, International Journal of Computer Vision, 83(2009), 274293.

[9] K. Kanatani, Y. Sugaya, Compact Fundamental Matrix Computation, In: 3rd Pacific Rim Symp. Image and Video Technology, Tokyo (2009), 179190. 


\begin{tabular}{|r|r|r|r|}
\hline points & $d_{\text {eight }}$ & $d_{C}$ & $d_{\text {rectification }}$ \\
\hline 1 & -0.0479 & -7.2225 & $-7.7626 .10^{-15}$ \\
\hline 2 & 0.008 & -3.149 & $1.8867 .10^{-14}$ \\
\hline 3 & 0.2082 & -5.9987 & $-1.0196 .10^{-14}$ \\
\hline 4 & 0.1773 & -6.0828 & $-1.2576 .10^{-15}$ \\
\hline 5 & 0.0652 & -2.1132 & $-3.6904 .10^{-14}$ \\
\hline 6 & 0.2827 & -7.1527 & $3.9944 .10^{-15}$ \\
\hline 7 & 0.0573 & 2.9146 & $4.6329 .10^{-13}$ \\
\hline 8 & -0.0348 & 2.7003 & $-4.9805 .10^{-13}$ \\
\hline 9 & -0.0425 & 3.6302 & $7.4464 .10^{-14}$ \\
\hline 10 & 0.0113 & 4.0314 & $1.1611 .10^{-12}$ \\
\hline
\end{tabular}

Table 2: The distance between point $M_{i}^{\prime}$ and its epipolar line

[10] F. Wenzel, R. Grigat, Parametrizations of the Essential and the Fundamental Matrix based on Householder Transformations, In: 10th International Workshop of Vision, Modeling, and Visualization, Erlangen (2005), 163-170.

[11] Z. Zhang, Determining the Epipolar Geometry and its Uncertainty, International Journal of Computer Vision, 27(2) (1998), 161-198.

[12] Y. Zheng, S. Sugimoto, M. Okutomi, A Practical Rank-Constrained EightPoint Algorithm for Fundamental Matrix Estimation, In: CVPR2013, Computer Vision Foundation, Ithaca, NY (2013), 1546-1553. 
